Article

\title{
Does Augmented Reality Affect Sociability, Entertainment, and Learning? A Field Experiment
}

\author{
Nina Savela ${ }^{1, *(\mathbb{D}}$, Atte Oksanen ${ }^{1} \mathbb{D}$, Markus Kaakinen ${ }^{2}$, Marius Noreikis ${ }^{3}$ and Yu Xiao ${ }^{3}$ \\ 1 Faculty of Social Sciences, Tampere University, 33100 Tampere, Finland; atte.oksanen@tuni.fi \\ 2 Institute of Criminology and Legal Policy, University of Helsinki, 00100 Helsinki, Finland; \\ markus.kaakinen@helsinki.fi \\ 3 Department of Communications and Networking, Aalto University, 02150 Espoo, Finland; \\ mnoreikis@yahoo.com (M.N.); yu.xiao@aalto.fi (Y.X.) \\ * Correspondence: nina.savela@tuni.fi
}

Received: 14 January 2020; Accepted: 15 February 2020; Published: 19 February 2020

\begin{abstract}
Augmented reality (AR) applications have recently emerged for entertainment and educational purposes and have been proposed to have positive effects on social interaction. In this study, we investigated the impact of a mobile, indoor AR feature on sociability, entertainment, and learning. We conducted a field experiment using a quiz game in a Finnish science center exhibition. We divided participants $(N=372$ ) into an experimental group (AR app users) and two control groups (non-AR app users; pen-and-paper participants), including 28 AR users of follow-up interviews. We used Kruskal-Wallis rank test to compare the experimental groups and the content analysis method to explore AR users' experiences. Although interviewed AR participants recognized the entertainment value and learning opportunities for AR, we did not detect an increase in perceived sociability, social behavior, positive affect, or learning performance when comparing the experimental groups. Instead, AR interviewees experienced a strong conflict between the two different realities. Despite the engaging novelty value of new technology, performance and other improvements do not automatically emerge. We also discuss potential conditional factors. Future research and development of AR and related technologies should note the possible negative effects of dividing attention to both realities.
\end{abstract}

Keywords: augmented reality; sociability; social interaction; entertainment; learning

\section{Introduction}

A new software technology combining physical and virtual realities by adding layers of digital information onto the physical world using suitable devices, such as wearables, has enabled new applications (e.g., Pokémon Go) and has introduced a novel way to communicate with others [1,2]. Changing the way people communicate and interact with each other, social media has been the most recent technological revolution that has impacted social interaction, entertainment, and learning [3]. These aspects are also apparent in perceived benefits that motivate people to use social media [4-6]. As new technology has the potential to permanently change the way we behave, besides user studies and product development, cutting-edge technology should be examined with regard to its implications on social interaction, sociability, entertainment, and learning.

Augmented reality (AR) applications enable people to interact with virtual objects overlaid in the physical environment and visible through digital screens. AR or mixed reality (MR) can be located in a continuum between real and virtual reality or seen as a combination of the two different elements $[7,8]$. As a result of the incoherent use of the terminology and commercial success of AR applications, previous researchers and other stakeholders have often referred to MR as AR, even though only some 
of the experts in the field use them as synonyms [8]. The suggested definitions of MR summarized by Speicher and colleagues [8] indicate that collaboration is one of the key aspects used in defining the concept. Piumsomboon and colleagues $[9,10]$, for example, studied the collaboration between the local AR user and remote virtual reality (VR) user.

Increasing amounts of AR and MR applications are being designed and introduced each year to the public and to various industry fields, such as education, health care, and maintenance, to name a few [11-13]. As an entertainment technology combined with a global positioning system, AR is best known to the public thanks to Pokémon GO, but new games and applications emerge, including Minecraft Earth and Harry Potter: Wizards Unite. Based on previous research, AR applications such as AR games could potentially enhance social communication and social interaction between people. Researchers have focused on the case of Pokémon Go and concluded that AR games have the potential for positive behavioral effects such as socialization [14]. Some games, not just AR games, incorporate more social and collaborative features to the gameplay than others, but even single-player games have been argued to have inherent social aspects [15]. Thus, augmented reality games could potentially foster sociability by gathering users together in a shared space and offering affordances for interacting with other users via options to comment, read, and reply to others. Mäyrä and colleagues [16] describe the single-player games in general, "knowledge of others playing the same game makes the game more social", which presumably concerns AR technology as well. Against this, it is interesting that a sense of social presence in MR technology has been found to positively affect visitor experiences in public places [17].

Entertainment value is also an important factor in itself and becomes even more relevant when investigating AR game outcomes in the context of recreational activities. More generally, gamification has combined research of technology and game design, motivation, and human-computer interaction, among others [18]. In a review of engagement in digital entertainment games, researchers categorized, for example, subjective experience, physiological responses, motives for playing, and impact of game on life satisfaction as aspects of engagement [19]. Recently, researchers studying AR identified hedonic and utilitarian as important aspects in AR adoption and found that consumers regard AR to be useful in tourism and education [20], and based on a literature review, AR had positive effects on engagement (e.g., in the museum context) [21]. Pervasive MR games are not without their challenges from hedonic and emotional perspectives. Enjoyment and engagement could be disrupted by, for example, awkwardness of playful behavior in public places [22]. Immersive games also impact the sense of presence [23], which could result in conflict between realities in the case of MR, but research on this remains scarce.

Based on a systematic literature review on the effects of computer games and serious games, there exist evidence about the effectiveness of game-based learning [24]. The authors concluded that "playing computer games is linked to a range of perceptual, cognitive, behavioral, affective and motivational impacts and outcomes" [24] (p. 661). For example, they found evidence on knowledge acquisition, motivational, and affective outcomes, but they noted the difficulties in defining learning outcomes [24]. In previous studies on AR and similar technologies, researchers have indeed focused heavily on their learning potential and have found similar findings than the aforementioned literature on game-based learning e.g., [25,26].

$\mathrm{Wu}$ and colleagues showed in their literature review on AR in education that research has emphasized the potential of AR to improve learning and aid in learning difficulties, but some challenges have also been noted regarding cognitive overload, lack of complex skills, and confusion between reality and fantasy [27]. In another systematic review, Bacca and colleagues demonstrate that the target group of most of the research on AR and learning has been students of bachelor level or lower, with only a couple targeting informal learners [28]. According to the authors, learning gains, motivation, and facilitated interaction and collaboration were the most reported advantages of AR [28]. Finally, in a more recent systematic literature review, researchers found mixed evidence about cognitive overload and called for future research on the effects of informal and ubiquitous learning, motivation, engagement, satisfaction, and interactions [25]. 
Researchers have highlighted social aspects such as social presence, social space, and sociability in learning in other technology contexts as well [29]. Kreijns and Kirschner [30] coupled educational affordance with social and entertaining affordances in a paper calling for consideration of these three perspectives on online collaborative learning. These aspects are also part of the uses and gratifications approach that seeks to explain the reasons and motivations behind media use with social, affective, and cognitive needs, among others [31]. However, our interest lies not in the end results of these motivations, such as perceived benefits and motivations to use, but, in the spirit of mass communication theory [32], in how the new AR technology is actually able to affect sociability, entertainment, and learning. This kind of research has not been done on AR technology before.

\section{This Study}

In this experimental study, we investigated how indoor AR technology influences users' sociability, entertainment, and learning in shared space. To test whether AR technology has an independent effect on the three aforementioned perspectives, we conducted a field experiment in a science center exhibition and designed an experiment comparing AR application participants to non-AR (a mobile application without AR features) and pen-and-paper participants. Based on previous research, AR technology could potentially have effects on sociability and communication with other players [14-16], as well as entertainment and learning-related consequences [20,21,25-28]. To investigate this further, this study has the following research questions:

1. Does augmented reality have an effect from the social perspective on (a) perceived sociability or social behavior of (b) leaving messages or (c) talking with others face-to-face?

2. Does augmented reality have an effect from the entertainment perspective on (a) excitement, (b) nervousness, (c) tiredness afterwards, (d) app satisfaction, or (e) exhibition enjoyment?

3. Does augmented reality have an effect from the educational perspective on learning performance?

More specifically, from the social perspective, we include aspects of perceived sociability and actual social behavior with other people. From the entertainment point of view, we examine perceived satisfaction with the exhibition and application, reported affects of excitement and nervousness during the experiment, and tiredness after the exhibition. Lastly, the educational perspective encompasses learning performance during the experiment. We compared all these aspects among three experimental groups that provided three different stimuli: AR application, an application without AR technology, and without smartphone application using only pen and paper. The experiment environment provides stimuli and opportunities for learning, entertainment, and social behavior through shared social space. Figure 1 shows the detailed study design.

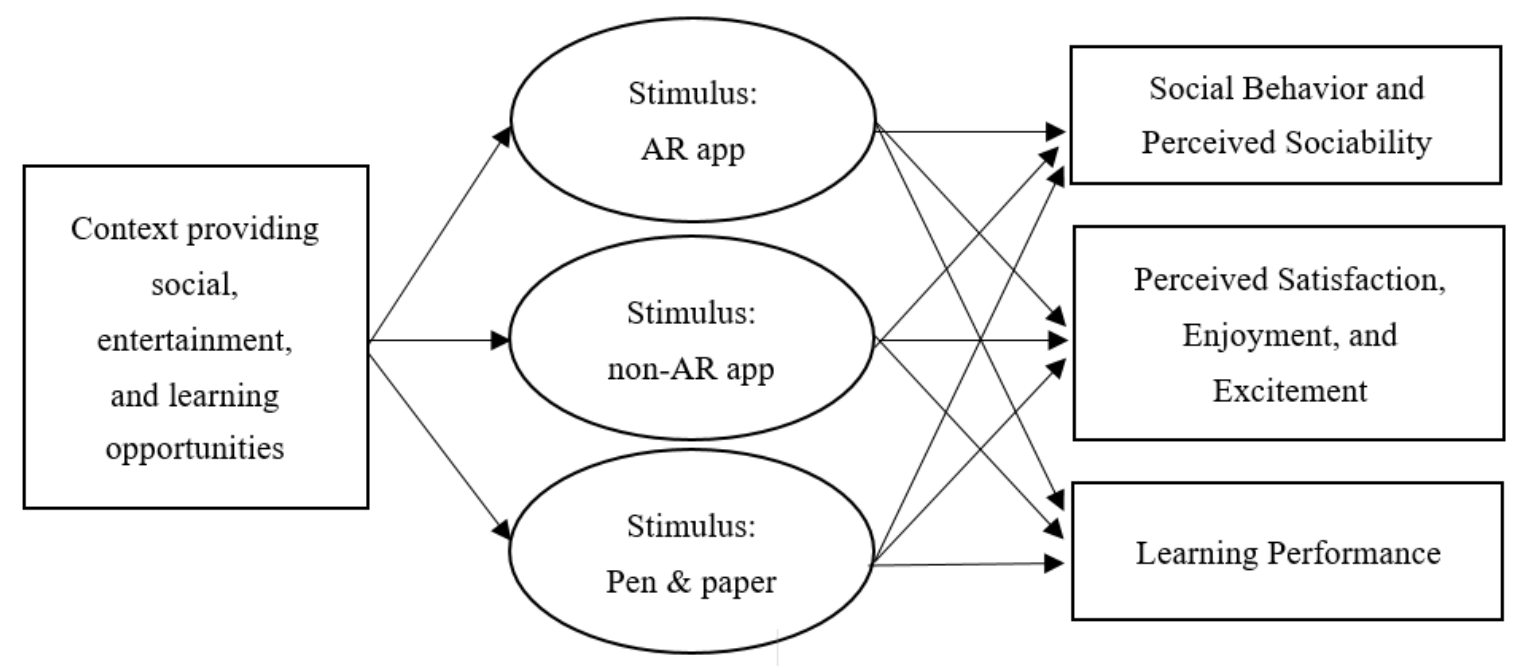

Figure 1. The study design. 


\section{Materials and Methods}

\subsection{Procedure}

We conducted a field experiment in the Finnish Heureka Science Centre to investigate the effects of AR technology on visitor experiences and behavior. Using the science center's own quiz related to biotechnology, we developed a quiz game for the AR app, app without AR feature (non-AR app), and pen-and-paper questionnaire. We invited the participants to participate in our study, and then we randomly assigned them to one of the three different conditions in the science center's main exhibition room, which presents multiple themes included in the quiz. All three conditions (AR, non-AR, and pen-and-paper) were carried out simultaneously and there was some variation depending on the day and number of visitors at the exhibition space. Participants received a small candy reward after the experiment.

The key feature of the AR app was for the visitors to interact with the quiz using AR. As they start, visitors look around the exhibition through their phone screens and discover the hidden quiz questions, represented as virtual floating question marks, scattered around the exhibition (see Appendix A). When a user approaches a question mark, the quiz questions are shown. Questions are placed near exhibits that are related to the question contents. Altogether, 15 questions were located around the room in eight different places. Therefore, one must tour the entire exhibition room to discover and answer all the quiz questions. Questions can be answered by tapping on one of the four possible answers. Once answered, the questions disappear, as well as the question mark hints. Users can keep track of the number of remaining questions. After all the questions are answered or if the users decide to quit the quiz, they are presented with a quiz score. Within the exhibition, users may also leave comments that will appear as an augmented 3-D object, placed on the museum floor. Other users can immediately see the comments left by others, read them, and post replies.

The non-AR app contains the same functionality as the AR app; however, a user solves a quiz on the phone's screen without AR interaction (see Appendix B). In contrast to the AR app, a non-AR app user can immediately browse through and answer all the quiz questions without the need to explore the exhibition. The quiz questions and scoring system are the same for both applications. Non-AR app users can also write comments next to a selected question and reply to comments left by other non-AR app users. They can also read the comments, but because opening the comments section immediately shows all the comments, we did not have a reliable way of determining which comments were actually read by a user and thus we have missing information about the read comments of non-AR app users.

Both applications were developed for the Android platform. To implement the AR feature in the AR app, we utilized image-base localization, the ARCore library by Google, and the Unity game engine by Unity Technologies. The non-AR app was developed using Google's Android Software Development Kit. The applications obtain questions and comments from a server and continuously send users' activity information to the server. The activity information indicates times when the quiz was started and finished, quiz scores, and read (for AR app only) and written comments. Furthermore, before and after the quiz, both applications ask users a series of questions that are also sent to the server. We stored all collected data in the database for later analysis.

We gave the other control group a pen-and-paper questionnaire with the quiz in the middle and questionnaire items before and after the quiz. We provided additional papers on a side desk next to the starting point and informed them that they could leave comments for other visitors to read and reply to. Similar to non-AR condition, we did not have a reliable way to detect if participants read any comments and thus, we have missing information about the read comments of pen-and-paper participants as well. All the experimental groups were offered two comments as a starting point: "You can leave comments and reply to comments left by others!" and "When you stand in a certain point of the room, you can see the Big Dipper." 


\subsection{Participants}

We collected the data in autumn 2018 from science center visitors $(N=372)$ who were aged 15-73 $(M=36.49, S D=11.71)$, and $55.95 \%$ of them were female. Of all the participants, 231 were given $A R$ applications, 71 participated with an application without AR technology, and 70 were handed pen and paper. Because our main interest was in the impact of AR technology, we recruited a larger number of AR participants to ensure enough information about the key phenomena. To assess the success of our randomization, we tested whether there were significant differences between our experimental groups in terms of background factors. We found that there were no differences between experimental groups in age, gender, extraversion trait, smartphone application self-efficacy, or previous experience with the exhibition. This means that the randomization was successful and found differences cannot be due to these background variables. In addition to the experiment, 28 users of AR application participated in a follow-up interview. All subjects gave their informed consent for inclusion before they participated in the study.

\subsection{Measures}

We measured the dependent variable of sociability with a same four-item instrument [33] before and after the game. This instrument was chosen because of its validated design of before and after measurement points. Participants answered statements on a scale from 1 (strongly disagree) to 7 (strongly agree): "Right now I feel social," "Right now I feel unsocial," "Right now I feel talkative," and "Right now I feel quiet." The sum variable of the items had a Cronbach's alpha of 0.86 before and 0.90 after the quiz. Average sum variable was used for the remainder after subtracting before from after values of perceived sociability. We measured the dependent variable related to social behavior by asking participants in the questionnaire whether they talked with other visitors and, if yes, whether they were unknown to them from before. We used these measures as dummy variables. In addition to the questionnaire items, we collected information about written comments and replies that were collected for every AR and non-AR app participant via the applications. The AR app also collected data about how many comments or replies were read by the users. After the field days, we collected papers left for pen-and-paper participants on a side desk for commenting.

The second set of dependent variables is related to the entertainment value and affects. Considering the reliable results of previous research in measuring immediate moment positive and negative affects [34,35], we asked all participants two questions in the middle of the quiz-that is, how excited or nervous they felt at the moment of filling the quiz. At the end of the questionnaire, participants also scored one statement about feeling tired after the exhibition. In addition to measuring perceived affects, we measured entertainment in the light of application and museum context. After the quiz, we asked them about their app satisfaction using three items (e.g., "the application I tested was fun to use") with a Cronbach's alpha of 0.83 . They also answered to two items of exhibition satisfaction (e.g., "the exhibition was interesting") with a Cronbach's alpha of 0.80 . We measured all items regarding entertainment value and affects on a scale from 1 to 7 . Average sum variable was used for the 3-and 2-item measures of app and exhibition satisfaction.

Thirdly, we measured participants' performance in the quiz. Scoring was based on how the science center scored its quizzes. If the correct answer to the question was "All of the above," a user received 3 points, and if only a single choice was selected when "All of the above" was a correct answer, a user received 1 point. After the first question, a user received 1 point for a correct answer and 0 for incorrect ones. In the analysis, we excluded those participants who did not finish the quiz. This information was collected with applications and calculated for pen-and-paper participants based on whether they answered the last question of the quiz.

We used a semi-structured interview method to collect a more in-depth account of user perceptions. The interview form contained 10 questions related to the experiences of the previous experiment (see Appendix C). The answers to the questions allowed us to examine the underlying reasons for the 
results of the experiment with the AR quiz application. Table 1 shows the descriptive statistics of all measures used in this study.

Table 1. Summary of Descriptive Statistics of the Study Variables $(N=372)$ : Frequency $(n)$, Percent $(\%)$, Mean $(M)$, Standard Deviation $(S D)$, Range, Number of Items in a Sum Variable, and Alpha $(\alpha)$.

\begin{tabular}{|c|c|c|c|c|c|c|c|}
\hline Measure & $n$ & $\%$ & $M$ & $S D$ & Range & $\begin{array}{c}n \text { of } \\
\text { Items }\end{array}$ & $\alpha$ \\
\hline Experimental groups & 372 & & & & & & \\
\hline $\begin{array}{l}\text { Experimental group (augmented reality [AR] } \\
\text { app participant) }\end{array}$ & 231 & 62.10 & & & & & \\
\hline Control group (non-AR app participant) & 71 & 19.09 & & & & & \\
\hline Control group (pen and paper participant) & 70 & 18.82 & & & & & \\
\hline Sociability before the experiment & 370 & & 4.76 & 1.19 & $1-7$ & 4 & 0.86 \\
\hline Sociability after the experiment & 370 & & 4.44 & 1.30 & $1-7$ & 4 & 0.90 \\
\hline Sociability after-before & 368 & & -0.32 & 1.18 & $-6-3.25$ & 8 & \\
\hline Talked with other visitors & 357 & & & & $0-1$ & 1 & \\
\hline No & 201 & 56.30 & & & & & \\
\hline Yes & 156 & 43.70 & & & & & \\
\hline Talked with unfamiliar visitors & 156 & & & & $0-1$ & 1 & \\
\hline No & 112 & 71.79 & & & & & \\
\hline Yes & 44 & 28.21 & & & & & \\
\hline Left comments & 372 & & & & $0-1$ & 1 & \\
\hline No & 346 & 93.01 & & & & & \\
\hline Yes & 26 & 6.99 & & & & & \\
\hline Excitement during experiment & 208 & & 4.34 & 1.24 & $1-7$ & 1 & \\
\hline Nervousness during experiment & 188 & & 3.30 & 1.66 & $1-7$ & 1 & \\
\hline Tiredness after the exhibition & 371 & & 3.61 & 1.49 & $1-7$ & 1 & \\
\hline App satisfaction & 302 & & 3.88 & 1.42 & $1-7$ & 3 & 0.83 \\
\hline Exhibition satisfaction & 371 & & 5.37 & 1.13 & $2-7$ & 2 & 0.80 \\
\hline Quiz score (from those who finished) & 155 & & 10.54 & 2.67 & $3-16$ & 1 & \\
\hline
\end{tabular}

\subsection{Analysis}

We used descriptive statistics, cross tabulation, $\chi^{2}$ test, Kruskal-Wallis equality of populations rank test, and Dunn's pairwise multiple comparison with Bonferroni corrections post hoc test to compare the experimental groups in measures of social behavior, perceived sociability, entertainment, and quiz performance. We chose non-parametric Kruskal-Wallis test by ranks over the ANOVA analysis of variance, because of the unequal sizes of the experimental groups and unequal variances based on Barlett's test for equal variances. For additional analysis we used the ordinary least squares (OLS) regression analysis and binary logistic regression methods, for which we report unstandardized regression coefficient $(B)$, odds ratio $(O R)$, and $p$ values. We performed all statistical analyses with Stata 12 and Stata 16. For Dunn's pairwise multiple comparisons, we used a Stata package programmed by Alexis Dinno [36].

We used the directed content analysis method [37] to explore AR application users' experiences of learning, entertainment, and social interaction, and also reasons for, for example, communicating (or not) with others. With this approach, we could use the research findings from the quantitative analysis to find relevant information related to the rationale of participants' behaviors.

\section{Results}

\subsection{Sociability and Social Behavior}

The results of the change in sociability before and after the quiz show that participants in all experimental groups felt, on average, less sociable after than before the quiz $(M=-0.32, S D=1.18$; see Table 2). Compared to AR users $(M=-0.32, S D=1.25)$, non-AR users felt even less sociable $(M=-0.52$, $S D=1.09)$, but the negative effect was smaller for pen-and-paper participants $(M=-0.08, S D=0.95)$. However, based on the Kruskal-Wallis rank test, the difference among the experimental groups was 
not statistically significant $\left(\chi^{2}[2, N=368]=5.64, p=0.060\right.$, with ties), except between the two control groups $\left(\chi^{2}[1, N=137]=5.99, p=0.014\right.$, with ties $)$.

Table 2. Means $(M)$, Standard Deviations $(S D)$, Frequencies (n), Rank Sum, and Dunn's Multiple Nonparametric Pairwise Post Hoc Test with Bonferroni Corrections for Differences in Dependent Variable of Sociability $(N=372)$.

\begin{tabular}{ccccccc}
\hline Sociability & $\boldsymbol{M}$ & $\boldsymbol{S D}$ & $\boldsymbol{n}$ & Rank Sum & 1. AR App & 2. Non-AR App \\
\hline Sociability after-before & -0.32 & 1.18 & 368 & & & \\
1. AR app & -0.32 & 1.25 & 231 & $42,473.00$ & & \\
2. Non-AR app & -0.52 & 1.09 & 71 & $11,707.00$ & 1.32 & $-2.37^{*}$ \\
3. Pen and paper & -0.08 & 0.95 & 66 & $13,716.00$ & -1.62 & \\
\hline$* p<0.05 . * * p<0.01 .^{* * *} p<0.001$. Note. The dependent variable: Perceived Sociability (After-Before the Quiz).
\end{tabular}

Table 3 shows the cross tabulation of talking with other visitors. We did not find statistically significant differences in talking to other visitors between the experimental groups, but the $\chi^{2}$ test result suggests that AR app users were slightly less likely to talk with others than the two control groups put together $\left(\chi^{2}[1]=5.97, p=0.015\right)$. In addition, from those participants who talked with someone, pen-and-paper participants were more likely to engage in a discussion with someone who was unknown to them from before compared to AR app users $\left(\chi^{2}[1]=14.41, p<0.001\right)$ and, to some extent, to non-AR app users $\left(\chi^{2}[1]=4.35, p=0.037\right)$.

Table 3. Cross Tabulation of Talking with Other Visitors by Experimental Groups $(N=372)$.

\begin{tabular}{ccccc}
\hline Measure & All Participants & AR App & Non-AR App & Pen and Paper \\
\hline $\begin{array}{c}\text { Talked with other } \\
\text { visitors }\end{array}$ & $357(100 \%)$ & $231(100 \%)$ & $71(100 \%)$ & $55(100 \%)$ \\
No & $201(56.3 \%)$ & $141(61.0 \%)$ & $34(47.9 \%)$ & $26(47.3 \%)$ \\
Yes & $156(43.7 \%)$ & $90(39.0 \%)$ & $37(52.1 \%)$ & $29(52.7 \%)$ \\
\hline $\begin{array}{c}\text { Talked with } \\
\text { unfamiliar visitors }\end{array}$ & $156(100 \%)$ & $90(100 \%)$ & $37(100 \%)$ & $29(100 \%)$ \\
No & $112(71.8 \%)$ & $73(81.1 \%)$ & $26(70.3 \%)$ & $13(44.8 \%)$ \\
Yes & $44(28.2 \%)$ & $17(18.9 \%)$ & $11(29.7 \%)$ & $16(55.2 \%)$ \\
\hline
\end{tabular}

Most participants did not leave any comments (see Appendix D). We provided pen-and-paper participants additional papers on a side desk next to the starting point for leaving comments, but they did not write anything down or reply to comments made by others. Non-AR app participants did not reply to any comments, and only four of them $(4 / 71,5.6 \%)$ left comments with the non-AR application. From the AR app participants, nearly every tenth left at least one comment (22/231, 9.5\%) or reply $(27 / 231,11.7 \%)$, and every other AR participant read at least one comment $(114 / 231,49.4 \%)$. Within the limitations of missing information of read comments by pen-and-paper and non-AR participants, the results show that AR users left more comments and replied more to other visitors' comments than the control groups.

In the follow-up interviews of AR users, some participants commented that AR could be suitable for social interaction among introverted people. However, they also reported conflict and disorientation in trying to pay attention to their surroundings and to other people while navigating the room and advancing in the quiz. Some reported not paying any attention to other people, and some perceived that other visitors were more of an annoyance because it was difficult to get close enough to the question spots and to move around the room, as presented in this comment: "I feel like I don't necessarily pay attention to other people, that I could even walk over someone. I was in there at the phone, but still it felt as if I wasn't here in this space" (Interviewee 11). One interviewee explicitly expressed not feeling sociable while participating in our experiment: “I definitely didn't feel myself sociable. I would have 
been more social without [the phone or app]" (Interviewee 5). Another interviewee did not think this app increases talking to other people because it was more of an individual task: "I feel that this app does not help interacting with others, at least not the one I used now. This was private activity" (Interviewee 9). Interviewees who reported having found comments and who talked about being engaged with the game were excited about finding the comments and question marks. Some reported not quite catching the point of them, and other times, people discussed the user interface of comments and questions.

\subsection{Entertainment}

Table 4 shows the results for excitement and nervousness during the quiz. Excitement during the quiz was moderately high for all participants $(M=4.28, S D=1.17)$. However, AR app users were less excited $(M=4.09, S D=1.36)$ than pen-and-paper participants $(M=4.68, S D=1.10)$, with non-AR participants being somewhere in the middle $(M=4.28, S D=1.17)$. The difference among experimental groups was statistically significant $\left(\chi^{2}[2, N=208]=9.73, p=0.008\right.$, with ties) based on the Kruskal-Wallis rank test. We found significant pairwise difference between AR users and pen-and-paper participants but not when comparing non-AR participants to other experimental groups. During the quiz, participants using phone applications were more nervous than pen-and-paper participants $(M=2.46, S D=1.43)$, with non-AR app users feeling most nervous $(M=4.35, S D=1.54)$ and AR app users somewhere between $(M=3.32, S D=1.48)$. The difference between the experimental groups and among all groups was statistically significant $\left(\chi^{2}[2, N=188]=39.11, p<0.001\right.$, with ties $)$ based on the Kruskal-Wallis rank test and Dunn's pairwise comparison with Bonferroni corrections post hoc test.

Table 4. Means (M), Standard Deviations (SD), Frequencies (n), Rank Sum, and Dunn's Multiple Nonparametric Pairwise Post Hoc Test with Bonferroni Corrections for Differences in Dependent Variables of Entertainment $(N=372)$.

\begin{tabular}{|c|c|c|c|c|c|c|}
\hline Entertainment & $M$ & $S D$ & $n$ & Rank Sum & 1. AR App & 2. Non-AR App \\
\hline Excitement during the quiz & 4.34 & 1.24 & 208 & & & \\
\hline 1. AR app & 4.09 & 1.36 & 80 & 7341.00 & & \\
\hline 2. Non-AR app & 4.28 & 1.17 & 60 & 6134.50 & -1.06 & \\
\hline 3. Pen and paper & 4.68 & 1.10 & 68 & 8260.50 & $-3.10^{* *}$ & -1.87 \\
\hline Nervousness during the quiz & 3.30 & 1.66 & 188 & & & \\
\hline 1. AR app & 3.32 & 1.48 & 66 & 6307.00 & & \\
\hline 2. Non-AR app & 4.35 & 1.54 & 54 & 6905.00 & $-3.29 * *$ & \\
\hline 3. Pen and paper & 2.46 & 1.43 & 68 & 4554.00 & $3.10 * *$ & $6.25 * * *$ \\
\hline Tiredness after the exhibition & 3.61 & 1.49 & 371 & & & \\
\hline 1. AR app & 3.54 & 1.42 & 231 & $41,995.50$ & & \\
\hline 2. Non-AR app & 3.34 & 1.52 & 71 & $11,905.50$ & 0.99 & \\
\hline 3. Pen and paper & 4.12 & 1.62 & 69 & $15,105.00$ & $-2.58 *$ & $-2.89 * *$ \\
\hline App satisfaction & 3.88 & 1.42 & 302 & & & \\
\hline 1. AR app & 3.78 & 1.37 & 231 & $33,401.50$ & & \\
\hline 2. Non-AR app & 4.20 & 1.56 & 71 & $12,351.50$ & $-2.49^{* *}$ & \\
\hline Exhibition satisfaction & 5.37 & 1.13 & 371 & & & \\
\hline 1. AR app & 5.33 & 1.11 & 231 & $41,776.50$ & & \\
\hline 2. Non-AR app & 5.36 & 1.24 & 71 & $13,207.00$ & -0.36 & \\
\hline 3. Pen and paper & 5.54 & 1.08 & 69 & $14,022.50$ & -1.53 & -0.96 \\
\hline
\end{tabular}

${ }^{*} p<0.05$. ${ }^{* *} p<0.01$. ${ }^{* * *} p<0.001$. Note. The dependent variables: excitement and nervousness during the quiz, app satisfaction, exhibition enjoyment, and tiredness after the exhibition.

Table 4 shows results also for tiredness after the exhibition, app satisfaction, and exhibition satisfaction. The reported tiredness after the exhibition was close to neutral for all participants $(M=3.61, S D=1.49)$. Participants using the pen-and-paper questionnaire felt more tired $(M=4.12$, $S D=1.62)$ compared to participants using phone applications, namely AR app users $(M=3.54$, 
$S D=1.42)$ and non-AR app users $(M=3.34, S D=1.52)$. The difference among the experimental groups was statistically significant $\left(\chi^{2}[2, N=371]=9.34, p=0.009\right.$, with ties) based on the Kruskal-Wallis rank test. Both app participants' satisfaction with the application was little above the middle of the scale. AR app users were slightly less satisfied with the application $(M=3.78, S D=1.37)$ than non-AR app users $(M=4.20, S D=1.56)$, the difference being statistically significant $\left(\chi^{2}[1, N=302]=6.19, p=0.013\right.$, with ties) based on the Kruskal-Wallis rank test. All participants were highly satisfied with the exhibition $(M=5.37, S D=1.13)$. Participants using pen and paper were most satisfied $(M=5.54, S D=1.08)$, followed by non-AR participants $(M=5.36, S D=1.24)$ and AR app users $(M=5.33, S D=1.11)$. The differences between the experimental groups and among all groups based on the Kruskal-Wallis rank test were not statistically significant $\left(\chi^{2}[2, N=371]=2.36, p=0.308\right.$, with ties).

AR interviewees reported high excitement in testing out new cutting-edge technology. Participants regarded the AR technology for the science center to be appropriate and complementary for the exhibition: "It felt quite natural and of course supports and expands this kind of exhibition environment well. I felt that the app was naturally well-suited to the exhibition" (Interviewee 13). Dissatisfied comments about app satisfaction were related to difficulties with some features of the app and learning how to use it: "At first it was a little confusing, but you get used to it" (Interviewee 12). One major theme in the interview data was the competitive side of the game and getting hooked in finding all the elements and finishing the game. This can be noted when one person mentioned being attracted to the comments and questions: "I noticed that the app attracted my attention and was searching for those others' comments and questions. I did like [the app]" (Interviewee 9).

\subsection{Learning Performance}

Table 5 shows the results regarding quiz performance. We excluded those who did not finish the quiz from this analysis. Based on the results, AR users received lower scores from the quiz $(M=8.59$, $S D=2.14)$ than the two control groups: pen-and-paper participants $(M=12.11, S D=2.05)$ and non-AR users $(M=10.75, S D=2.56)$. The difference among the experimental groups was statistically significant $\left(\chi^{2}[2, N=155]=47.36, p<0.001\right.$, with ties). Dunn's pairwise comparison with Bonferroni corrections post hoc test shows a statistically significant result for all pairwise comparisons. We did additional regression analysis and found that AR users who reported higher satisfaction on the app were more likely to finish the quiz $(O R=1.65, p<0.001)$. In turn, participants who finished the quiz had higher quiz scores $(B=7.41, p<0.001)$.

Table 5. Means (M), Standard Deviations (SD), Frequencies (n), Rank Sum, and Dunn's Multiple Nonparametric Pairwise Post Hoc Test with Bonferroni Corrections for Differences in Dependent Variable of Learning Performance $(N=372)$.

\begin{tabular}{ccccccc}
\hline $\begin{array}{c}\text { Learning } \\
\text { Performance }\end{array}$ & $\boldsymbol{M}$ & $\boldsymbol{S D}$ & $\boldsymbol{n}$ & Rank Sum & 1. AR App & 2. Non-AR App \\
\hline Quiz score & 10.54 & 2.67 & 155 & & & \\
1. AR app & 8.59 & 2.14 & 49 & 2170.50 & & \\
2. Non-AR app & 10.75 & 2.56 & 53 & 4378.50 & $-4.34^{* * *}$ & \\
3. Pen \& paper & 12.11 & 2.05 & 53 & 5541.00 & $-6.82^{* * *}$ & $-2.53^{*}$ \\
\hline
\end{tabular}

${ }^{*} p<0.05 .{ }^{* *} p<0.01 .{ }^{* * *} p<0.001$. Note. The dependent variable: Learning performance based on quiz score.

Interviews with AR users after the experiment revealed that AR participants assumed a high potential of AR technology in teaching and providing engaging learning tools, as was apparent in one comment:

Definitely, I can see it could support learning, mine and others as well. It was especially nice that it was a part of the exhibition space. I also liked the scoring. If those were utilized more, I think it would have supported the learning more. (Interviewee 16). 
High engagement in the competitive features of finding and answering all the questions was a common theme. However, some interviewees had doubts that AR technology could actually improve learning and performance outcomes: "I believe using augmented reality was more meaningful, but I don't believe it increased learning" (Interviewee 10). The previously reported results of comparing AR users' quiz performance to the two control groups provide support for this suspicion, but the interviews still highlight the potential entertainment value in learning, which cannot be measured by performance.

\section{Discussion}

In this experimental field study in the science center environment, we investigated the social, entertaining, and educational aspects of indoor AR technology. Our findings from comparing the AR app to the app without the AR feature and pen-and-paper condition did not confirm a positive impact of AR technology toward perceived sociability, social communication, entertainment, or learning performance, although we detected some evidence of engaging novelty value and increased commenting behavior. In addition, we found that $\mathrm{AR}$ users experienced some discomfort in dividing their attention between the virtual and physical world.

We did not find evidence on the positive impact of AR technology on perceived sociability. Our findings suggest that AR app users are slightly less likely to talk to other people outside the application compared to participants using non-AR app or pen-and-paper, and less likely to talk with unfamiliar people compared to the latter. However, based on the descriptive analysis of comments left, read, and replied to, AR users' commenting activity was higher than that of the control groups. One reason for this could be that the AR application provided easily locatable and accessible opportunities to communicate with others through technology, which increased the amount of comments compared to non-AR app users and pen-and-paper participants. It should be noted, however, that the commenting options between the three groups were fundamentally different in regard to user interface and realistic commenting opportunities with pen and paper. Considering these as qualities of the three features used in the experiment, we found some tentative evidence that the AR feature has the potential to increase social behavior inside the app. To conclude, we found some potential for AR to increase social behavior within the app but did not find positive effects on social behavior outside the app or on perceived sociability. Our results were somewhat different compared to what other researchers have argued regarding social aspects of single-player games $[15,16]$ and suggesting that AR has a positive impact on sociability [14].

From the entertainment perspective, applications seem to arouse less excitement and more nervousness than the traditional pen-and-paper survey method, yet users of applications were less tired after the exhibition. One possible explanation is the learning curve of new and unfamiliar technology, as well as the feeling of uncertainty and not being in control. Filling out a survey and a quiz via a smartphone application can still perhaps be quicker and less arduous than circling answers on a paper form. Participants of the non-AR app were more satisfied with their app than AR app users. The unfamiliarity of AR applications could contribute to app satisfaction. We found no differences in exhibition satisfaction among the experimental groups; thus, none of the methods increased or decreased exhibition satisfaction. In comparing AR to other conditions, we did not find the positive effects of AR on the aspects of entertainment suggested in the literature [20,21], although a lower level of tiredness in AR participants could imply higher engagement or ease of using the AR application.

From the perspective of learning, we considered learning performance via measuring the participants' quiz scores. AR users scored significantly lower compared to the control groups, despite many AR interviewees reporting being engaged and ambitious toward the gamified quiz. Based on the interviews, some AR users also thought it could stimulate learning. The AR app was thought to offer novelty and meaningfulness to the learning experience, and this does not necessarily increase learning or performance compared to other means of learning. As an additional analysis, however, we found that those AR participants that were satisfied with the application were more likely to finish the quiz and, in turn, performed better in the quiz. The findings show that implementing new technology or gamification does not always enhance performance, although their effectiveness is supported by 
previous research on game-based learning [24] and learning with AR or similar technologies [25-28]. Our study contributes to the research on educational uses of AR by offering valuable information from an empirical experiment on informal learners in the context of recreation [28].

The interviews revealed that AR users saw a lot of potential for AR technology as a learning tool for education and as a complementary feature in the science center and other attractions. However, the interviewees saw less potential in AR for social behavior. Interview results show that AR technology causes a strong conflict between the two different environments: the reality seen through the technology enhanced with virtual elements and the reality shared with people around the same room. For these AR users, the latter social environment was seen as a disruption while trying to focus on the task and the reality seen through the AR application. Thus, the pervasive AR games pose some hedonic and emotional challenges for the users. The findings were in line with previous research on the perceived awkwardness of playful behavior in public places [22] and the immersive nature of games that impacts the sense of presence [23]. Although mixed results regarding cognitive overload exist [25,27], perceived conflict between realities using AR or similar technology has not been addressed in previous research and should be investigated further.

\subsection{Strengths and Limitations}

Our study was limited to certain applications and a science center context, which should be noted when interpreting the results. Our application was fairly simple, and some participants may have had high expectations based on commercial applications with advanced design processes such as Pokémon Go that has been developed by Niantic, Nintendo, and The Pokémon Company. On the other hand, we consider that the simple design of our application was the strength of this study. It made the AR condition more similar to other experimental conditions (i.e., app without AR feature and pen-and-paper), which were also simple by design, mainly based on the exhibition quiz. In addition, we were able to show the difficulties that users face when using an app for the first time. However, this also means that our experiment was limited to one user experience at one point in time. The negative effects could arguably disappear after people become more familiar with AR technology. For this reason, future researchers should aim to understand how the use of AR apps develops over time and whether AR technology has a social, entertaining, or educational impact when using the technology for a longer period of time.

Lastly, the possible impact of the lack of something in common with others and the self-awareness caused by the experimental nature of the situation should also be considered as conditional factors. A science exhibition itself is not always considered a social space, and participants may have reservations in commenting, as they are not used to this during exhibitions. It might be more natural and relevant for users to participate outside the experimental environment, where they can leave and read comments from people of their own social network or from people with common interests and goals, compared to a wide and unfamiliar group of science center visitors. In that case, the social features could perhaps be more effective in generating social interaction. Yet, by not explicitly encouraging participants to interact but informing them on the communication facilities available, our experimental setting allowed us to analyze whether the mere communicative facilities of AR technology (not the used encouragement or incentive) can foster social interaction and ensured the comparability between the different experimental conditions. The effect of different encouragement strategies would still be worthy of future investigations.

\subsection{Conclusions}

Based on the field experiment, our conclusion is that an AR game does not necessarily provide improvements to learning performance or social interaction, at least in communicating outside the technology. Phone applications seem to cause more nervousness and less excitement but also less tiredness than using the traditional survey method of pen and paper. Our results indicate that AR does have entertainment value as a novel technology, but it poses some challenges for people to be 
present and devoted to both realities at the same time. Thus, the results suggest that the positive effects of augmented reality are not self-evident. On the contrary, utilizing AR technology in natural environments can produce unexpected and even negative results. Given our findings, more field experiments on AR technology is needed. Future research and the development of AR technology should consider the negative emotions related to choosing between the two realities and the learning curve of using AR technology when high performance or learning outcomes are desired.

Author Contributions: Conceptualization, N.S., A.O., M.K., M.N. and Y.X.; methodology, N.S., A.O. and M.K.; software, M.N. and Y.X.; validation, N.S., A.O., M.K., M.N. and Y.X.; formal analysis, N.S. and A.O.; investigation, N.S. and M.N.; resources, A.O. and Y.X.; data curation, N.S. and M.N.; writing-original draft preparation, N.S.; writing-review and editing, N.S., A.O., M.K., M.N. and Y.X.; visualization, N.S. and M.N.; supervision, A.O. and Y.X.; project administration, A.O. and Y.X. All authors have read and agreed to the published version of the manuscript.

Funding: This research received no external funding.

Acknowledgments: We acknowledge the resources and support to this research provided by the Finnish Heureka Science Centre, Aalto University, University of Helsinki, and Tampere University.

Conflicts of Interest: The authors declare no conflicts of interest.

\section{Appendix A}

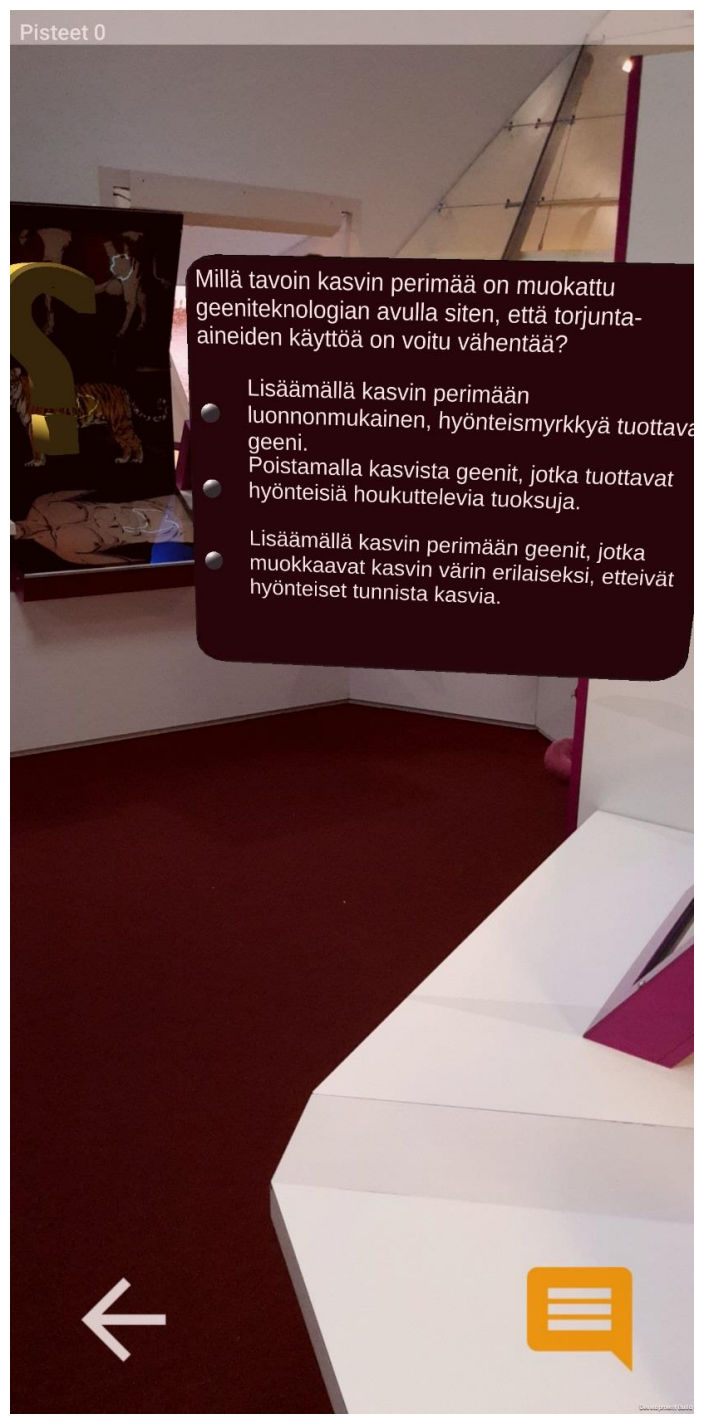

Figure A1. A Screenshot of the AR App Game Mode. 


\section{Appendix B}

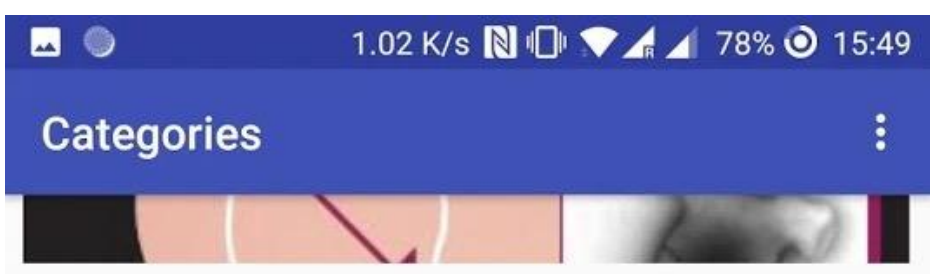

\section{Biotechnology in food preservation (The Wind in the Bowels)}

Biotechnology has been used in food preservation and preparation for thousands of years, since 4000-7000 BCE. Lactic acid bacteria have been used for fermentation, for example, to turn milk into yoghurt or viili, the traditional Scandinavian fermented milk product.

Answered $2 / 2$

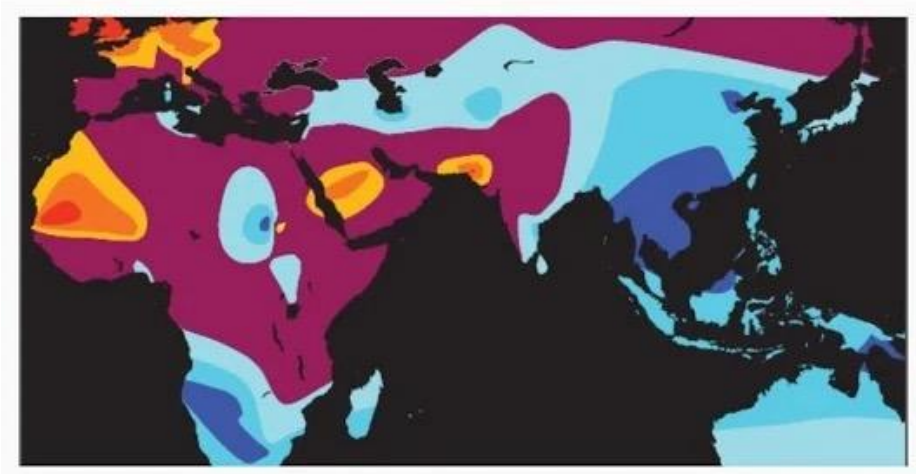

Biotechnology in our diet (The Wind in the Bowels)

Lactose intolerance is a common condition all over the world. Lactose-intolerant people can eat dairy products without stomach problems if the lactose is first removed from the products. Lactose, a sugar found in milk products, can be broken down with enzymes.

Answered $2 / 2$

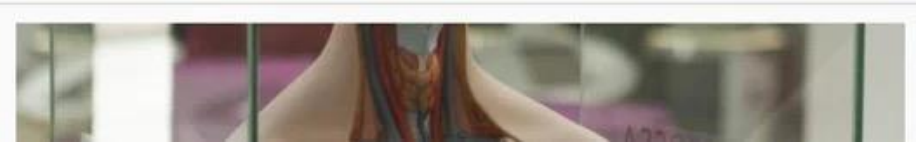

Figure A2. A Screenshot of the Non-AR App Game Mode.

\section{Appendix C}

\section{The Interview Form}

(1) What was your experience of the application?

(2) Did the experience here differ from other museum exhibition experiences you have? How?

(3) How did the application influence your behavior in the exhibition space?

(4) What kind of cell phone application would make the exhibition more interesting?

(5) Do you think you are outgoing?

(6) Do augmented reality applications suit you?

(7) Have you used mobile applications with augmented reality before?

(8) What kind of learning experience was it for you to answer the quiz? 
(9) Do you think augmented reality technology had anything to do with your learning?

(10) Did you finish the quiz? Do you think augmented reality technology had anything to do with your motivation to finish the quiz?

\section{Appendix D}

Table A1. Commenting Activity by AR Users and Non-AR Users: Frequencies of Comments Left, Replied, and Read $(N=372)$.

\begin{tabular}{ccccccccc}
\hline Amount of comments left: & 0 & 1 & 2 & 3 & 4 & 5 & 6 & Total \\
\hline 1. AR app & 209 & 15 & 3 & 2 & 1 & & 1 & 231 \\
2. Non-AR app & 67 & 2 & 2 & & & & & 71 \\
3. Pen and paper & 70 & & & & & & & 70 \\
\hline Amount of replies made: & 0 & 1 & 2 & 3 & 4 & 5 & $6<$ & Total \\
\hline 1. AR app & 204 & 17 & 6 & 2 & 2 & & & 231 \\
2. Non-AR app & 71 & & & & & & 71 \\
3. Pen and paper & 70 & & & & & & & 70 \\
\hline Amount of comments read: & 0 & 1 & 2 & 3 & 4 & 5 & $6<$ & Total \\
\hline 1. AR app & 117 & 30 & 30 & 18 & 17 & 10 & 9 & 231 \\
\hline
\end{tabular}

\section{References}

1. Bastug, E.; Bennis, M.; Médard, M.; Debbah, M. Toward interconnected virtual reality: Opportunities, challenges, and enablers. IEEE Commun. Mag. 2017, 55, 110-117. [CrossRef]

2. Sherman, W.R.; Craig, A.B. Understanding Virtual Reality: Interface, Application, and Design, 2nd ed.; Morgan Kaufmann: Cambridge, MA, USA, 2018.

3. Van Dijck, J.; Poell, T. Understanding social media logic. Media Commun. 2018, 1, 2-14. [CrossRef]

4. Kim, Y.; Sohn, D.; Choi, S.M. Cultural difference in motivations for using social network sites: A comparative study of American and Korean college students. Comput. Hum. Behav. 2011, 27, 365-372. [CrossRef]

5. Lin, K.Y.; Lu, H.P. Why people use social networking sites: An empirical study integrating network externalities and motivation theory. Comput. Hum. Behav. 2011, 27, 1152-1161. [CrossRef]

6. Whiting, A.; Williams, D. Why people use social media: A uses and gratifications approach. Qual. Mark. Res. Int. J. 2013, 16, 362-369. [CrossRef]

7. Ohta, Y.; Tamura, H. Mixed Reality: Merging Real and Virtual Worlds; Springer Publishing: New York, NY, USA, 2014.

8. Speicher, M.; Hall, B.D.; Nebeling, M. What is mixed reality? In Proceedings of the 2019 CHI Conference on Human Factors in Computing Systems, CHI '19, Glasgow, UK, 4-9 May 2019. [CrossRef]

9. Piumsomboon, T.; Day, A.; Ens, B.; Lee, Y.; Lee, G.; Billinghurst, M. Exploring enhancements for remote mixed reality collaboration. In Proceedings of the SA '17 SIGGRAPH Asia 2017 Mobile Graphics \& Interactive Applications, Bangkok, Thailand, 27-30 November 2017. [CrossRef]

10. Piumsomboon, T.; Lee, G.A.; Hart, J.D.; Ens, B.; Lindeman, R.W.; Thomas, B.H.; Billinghurst, M. Mini-me: An adaptive avatar for mixed reality remote collaboration. In Proceedings of the $2018 \mathrm{CHI}$ Conference on Human Factors in Computing Systems, CHI '18, Montréal, QC, Canada, 21-26 April 2018. [CrossRef]

11. Gavish, N.; Gutiérrez, T.; Webel, S.; Rodríguez, J.; Peveri, M.; Bockholt, U.; Tecchia, F. Evaluating virtual reality and augmented reality training for industrial maintenance and assembly tasks. Interact. Learn. Environ. 2015, 23, 778-798. [CrossRef]

12. Kurilovas, E. Evaluation of quality and personalisation of VR/AR/MR learning systems. Behav. Inf. Technol. 2016, 35, 998-1007. [CrossRef]

13. Rauschnabel, P.A.; Rossmann, A.; tom Dieck, M.C. An adoption framework for mobile augmented reality games: The case of Pokémon Go. Comput. Hum. Behav. 2017, 76, 276-286. [CrossRef]

14. Serino, M.; Cordrey, K.; McLaughlin, L.; Milanaik, R.L. Pokémon Go and augmented virtual reality games: A cautionary commentary for parents and pediatricians. Curr. Opin. Pediatr. 2016, 28, 673-677. [CrossRef] 
15. Stenros, J.; Paavilainen, J.; Mäyrä, F. Social interaction in games. Int. J. Arts Technol. 2011, 4, $342-358$. [CrossRef]

16. Mäyrä, F.; Stenros, J.; Paavilainen, J.; Kultima, A. From social play to social games and back: The emergence and development of social network games. In New Perspectives on the Social Aspects of Digital Gaming: Multiplayer 2; Kowert, R., Quandt, T., Eds.; Routledge: Abingdon, UK, 2017; pp. 11-32.

17. Jung, T.; tom Dieck, M.C.; Lee, H.; Chung, N. Effects of virtual reality and augmented reality on visitor experiences in museum. In Information and Communication Technologies in Tourism; Inversini, A., Schegg, R., Eds.; Springer: Cham, Switzerland, 2016; pp. 621-635. [CrossRef]

18. Deterding, S. The lens of intrinsic skill atoms: A method for gameful design. Hum. Comput. Interact. 2015, 30, 294-335. [CrossRef]

19. Boyle, E.A.; Connolly, T.M.; Hainey, T.; Boyle, J.M. Engagement in digital entertainment games: A systematic review. Comput. Hum. Behav. 2012, 28, 771-780. [CrossRef]

20. Grzegorczyk, T.; Sliwinski, R.; Kaczmarek, J. Attractiveness of augmented reality to consumers. Technol. Anal. Strateg. 2019, 31, 1257-1269. [CrossRef]

21. Berryman, D.R. Augmented reality: A review. Med. Ref. Serv. Q. 2012, 31, 212-218. [CrossRef] [PubMed]

22. Montola, M. A ludological view on the pervasive mixed-reality game research paradigm. Pers. Ubiquitous Comput. 2011, 15, 3-12. [CrossRef]

23. Cummings, J.J.; Bailenson, J.N. How immersive is enough? A meta-analysis of the effect of immersive technology on user presence. Media Psychol. 2016, 19, 272-309. [CrossRef]

24. Connolly, T.M.; Boyle, E.A.; MacArthur, E.; Hainey, T.; Boyle, J.M. A systematic literature review of empirical evidence on computer games and serious games. Comput. Educ. 2012, 59, 661-686. [CrossRef]

25. Akçayır, M.; Akçayır, G. Advantages and challenges associated with augmented reality for education: A systematic review of the literature. Educ. Res. Rev. 2017, 20, 1-11. [CrossRef]

26. Garzón, J.; Pavón, J.; Baldiris, S. Systematic review and meta-analysis of augmented reality in educational settings. Virtual Real. 2019, 23, 447-459. [CrossRef]

27. Wu, H.K.; Lee, S.W.Y.; Chang, H.Y.; Liang, J.C. Current status, opportunities and challenges of augmented reality in education. Comput. Educ. 2013, 62, 41-49. [CrossRef]

28. Bacca, J.; Baldiris, S.; Fabregat, R.; Graf, S. Augmented reality trends in education: A systematic review of research and applications. Educ. Technol. Soc. 2014, 17, 133-149.

29. Kreijns, K.; Kirschner, P.A.; Jochems, W.; Van Buuren, H. Determining sociability, social space, and social presence in (a) synchronous collaborative groups. CyberPsychol. Behav. 2004, 7, 155-172. [CrossRef] [PubMed]

30. Kreijns, K.; Kirschner, P.A. Extending the SIPS-Model: A research framework for online collaborative learning. In European Conference on Technology Enhanced Learning; Pammer-Schindler, V., Pérez-Sanagustín, M., Drachsler, H., Elferink, R., Scheffel, M., Eds.; Springer: Cham, Switzerland, 2018; pp. 277-290. [CrossRef]

31. Katz, E.; Blumler, J.G.; Gurevitch, M. Uses and gratifications research. Public Opin. Q. 1973, 37, 509-523. [CrossRef]

32. McQuail, D. Mass Communication Theory, 5th ed.; Sage: London, UK, 2005.

33. Whelan, D.C.; Zelenski, J.M. Experimental evidence that positive moods cause sociability. Soc. Psychol. Pers. Sci. 2012, 3, 430-437. [CrossRef]

34. Laurent, J.; Catanzaro, S.J.; Joiner, T.E., Jr.; Rudolph, K.D.; Potter, K.I.; Lambert, S.; Osborne, L.; Gathright, T. A measure of positive and negative affect for children: Scale development and preliminary validation. Psychol. Assess. 1999, 11, 326-338. [CrossRef]

35. Watson, D.; Clark, L. The PANAS-X: Manual for the Positive and Negative Affect Schedule-Expanded Form; The University of Iowa: Iowa City, IA, USA, 1999. [CrossRef]

36. Dinno, A. Nonparametric pairwise multiple comparisons in independent groups using Dunn's test. Stata J. 2015, 15, 292-300. [CrossRef]

37. Hsieh, H.F.; Shannon, S.E. Three approaches to qualitative content analysis. Qual. Health Res. 2005, 15, 1277-1288. [CrossRef]

(C) 2020 by the authors. Licensee MDPI, Basel, Switzerland. This article is an open access article distributed under the terms and conditions of the Creative Commons Attribution (CC BY) license (http://creativecommons.org/licenses/by/4.0/). 